\title{
KULTURELLE UNTERSCHIEDE
}

\section{Lutz HUTH* Sevgi GÜREZ ${ }^{* *}$}

\begin{abstract}
Differences between cultures/civilizations have decisive influence on the communication between their members. These differences, however,are situated in differing layers between observable behaviour and background phenomena like tradition or religion. It is the first purpose of this article to suggest a pragmatic differentiation on the scale $<$ concrete/abstract $>$. Pivotal relevance will be ascribed to the level of cultural dispositions, being a continuation of HOFSTEDE's mental programs, since this is the level of action orientation.

A further hypothesis will be that some cultural differences are rooted in history (genuine differences) while others are due to different levels of modernisation of society. Modernisation is meant in the sense of LUHMANN's system theory as social evolution from a horizontal to a hierarchichal and finally to a functional differentiation of society; this implies neither any concept of teleology nor any value-judgement. Methodically, it is presumed that differences between cultures / civilisations depend upon the kind and degree of social differentiation; only in the case of failure to support this it should be assumed that we are faced with (inexplicable) genuine differences.

Under these premises there will be a classification of cultural differences in accordance with the theory of communicative functions as presented by BÜHLER. To exemplify thre theoretical claims of this article some correspondences between the different layers will be presented, including observable communicative behaviour.

To conclude, the point will be raised which consequences may - on the basis of this analysis -be drawn in respect to different cultures under conditions of globalisation.

\section{Özet}

Kültürlerarası farklılıklar, farklı kültür mensupları arasındaki karşılıklı etkileşim, yani iletişim sürecinde belirleyici ve yönlendirici rol oynarlar. Bu farklılıkların kaynağı gelenekler ve dinler gibi çok geniş bir yelpazeyi oluşturur. Bu husus göz önünde tutularak bu çalışmada daha pratik bir çerçeve benimsenerek kültürel farklılaşma konusu "somutsoyut" ölçeği bazında ele alınmıştır. Bu bağlamda Hofstede'nin zihinsel programlar
\end{abstract}

${ }^{*}$ Prof. Dr. Dr. h. c., Universität der Künste Berlin

${ }^{* *}$ Araş. Gör., Universität der Künste Berlin 
olarak geliştirdiği ve güncelleştirdiği kültürel davranış örüntüleri, merkezî bir rol oynamaktadır. Zira bunlar, eylem oriyentasyonunun düzeyi olarak gerekçelendirilmektedir.

$\mathrm{Bu}$ konu ile ilgili bir diğer varsayıma göre, kültürel farklılıklardan bazılarının kökenlerinin tarihsel olduğu; bazılarının ise, modernleşme sürecinin farklı aşamalarının bir sonucu olduğu ifade edilmektedir. Luhmann'ın geliştirdiği sistem kuramı çerçevesinde modernleşme, sosyal bir evrim olarak nitelendirilmektedir. Bu süreç içerisinde toplum, yatay farklılaşmadan önce, dikey yani hiyerarşik, daha sonra da işlevsel bir farklılaşmaya geçiş yapar. Bu evrim ile, ne dini ne de herhangi diğer bir değerlendirme söz konusu edilmez.

$\mathrm{Bu}$ çalışmada hareket noktamızı, kültürel farklılıkların toplumsal farklılaşmanın biçimi ve aşamasından kaynaklandığı; şayet bunun geçerliliği kanıtlanamaz ise, söz konusu farklılıkların tarihsel olduğu düşüncesi oluşturmaktadır.

Buna göre kültürel farklılıklar, Bühler'in iletişimin işlevi veya modeli temel alınarak sınıflandırılmaktadır. Bu bağlamda, iletişimsel davranışları da içeren farklı düzeyler arasındaki çıkarımlara yönelik ve daha çok örnekleme amacıyla önermelerde bulunulmaktadır.

Bu çalışma, "küreselleşme olgusu 1şığında analizimizden her bir kültüre yönelik ne gibi sonuçların ortaya çıkabileceği?” sorusunun tartışılması ile son bulmaktadır.

\section{Problem Und Ausgangslage}

Unterschiede zwischen Kulturen finden seit gut zwei Jahrzehnten in den verschiedensten Zusammenhängen zunehmend Beachtung: in der Wirtschaft, der Politik, in Wissenschaft und Schule/Bildung, im militärischen Bereich, Dafür gibt es einige Gründe, die auf der Hand liegen: die transnationalen wirtschaftlichen Verflechtungen; engere politische Kontakte, in denen die kulturellen Bindungen der Beteiligten nicht vernachlässigt werden dürfen; die weltweiten Migrationsbewegungen, die dauerhaft enge und alltägliche Kontakte zwischen Angehörigen verschiedener Kulturen zur Folge haben; militärische Aktionen mit Beteiligten aus unterschiedlichen Kulturen in Einsatzgebieten, die wiederum anderen Kulturen angehören; die Entwicklung von Transporttechnologien, die - etwa im Tourismus zahlreiche kurzfristige Kontakte zwischen Mitgliedern verschiedener Kulturen ermöglicht; schließlich die Entwicklung, Verbreitung und intensive Nutzung von Kommunikationstechnologien, über die weltweit beliebige Kontakte hergestellt werden. Der Ausdruck Globalisierung bietet sich als offene Sammelbezeichnung für diese und weitere Phänomene an. 
Ein zweites Ursachenbündel wird aus der Tatsache hergeleitet, daß mit dem Ende des Kalten Krieges die ideologisch begründete Globaldifferenzierung <Ost/West $>$ und die damit gegebene politische Unterscheidung zwischen Freud und Feind entfallen ist. Solange diese Unterscheidung bestimmend war, überlagerte sie historisch gewachsene kulturelle Unterschiede, die vielfach als überholt erschienen, dann aber nach 1989/92 - zur Überraschung vieler wieder Aktualität gewannen; der Balkan, speziell Jugoslawien, wie auch die südlichen Staaten der ehemaligen Sovietunion sind geläufige Beispiele für diesen Prozeß.

Kulturbedingte Unterschiede verdienen, daß man sich systematisch mit ihnen beschäftigt, weil sie die Beziehungen zwischen Angehörigen unterschiedlicher Kulturen - speziell die Kommunikation zwischen ihnen - belasten können. Als Problem ist dies durchaus bekannt; und es fehlt nicht an Untersuchungen darüber und Vorschlägen dazu, die damit verbundenen Schwierigkeiten $\mathrm{zu}$ minimieren. Wo es nicht um Sprachprobleme geht, die sich durch Sprachunterricht mindern lassen, geraten derartige Arbeiten schnell ins Appellantenhafte, wo das Beschwören von Verständnis und Toleranz unter der Prämisse, daß alle Menschen gleich sind, an die Stelle der analytischen Auseinandersetzung tritt. Seltener sind dagegen Arbeiten, die sich systematisch um Klärung bemühen und versuchen, die alltäglichen Probleme im Rahmen eines umfassenderen Konzeptes zu behandeln.

\subsection{Kultur - Pragmatisch}

Wenn hier von Kultur die Rede ist, dann - ganz pragmatisch - im Sinne von Landeskultur; und - nochmals vereinfachend - verstehen wir Landeskultur zunächst als die in einem Staat verbreitete Kultur. Wir übergehen dabei regionale wie soziologisch bestimmbare Binnenkulturen (z.B. Jugendkultur, Kulturen einzelner Berufsstände, \&c.). Als Arbeitsdefinition von Kultur wählen wir für die vorliegende Fragestellung die Menge der in einer sozialen Gruppe wechselseitig unterstellbaren und damit anschlußfähigen Erwartungen; und bei einer gewissen Verallgemeinerung wird man sich darauf einlassen können, daß unter diesem Aspekt Länder/Staaten Einheiten bilden, die sich erkennbar voneinander unterscheiden. Auch wenn bei einer gewissen Vertrautheit Unterschiede zwischen Schotten, Engländern aus dem Norden und solchen in den Home Counties unübersehbar sind; der Ausdruck British 
umfaßt eine Menge von Verhaltenserwartungen, die sich von denen auf dem Kontinent abgrenzen lassen.

Ferner: der Begriff von Kultur wird hier jeweils auf eine Einzelkultur in allen ihren Erscheinungsformen bezogen; er ist nicht beschränkt auf das, was üblicherweise als Hochkultur bezeichnet wird - also Kunst oder die Verhaltensformen der oberen sozialen Schichten.

Wenn wir Kultur als die Menge anschlußfähiger Verhaltenserwartungen fassen, so ist damit das Problem beim Zusammenkommen von Angehörigen verschiedener Kulturen umrissen: Werden Erwartungen nicht erfüllt, so bricht die Kommunikation zusammen oder verläuft in einer nicht erwarteten Richtung, um dann zu versanden oder abgebrochen zu werden.

\subsubsection{Kulturanalyse Als Analyse Von Unterschieden}

Kultur, verstanden als Einzel-, hier als Landeskultur, kommt überhaupt erst im Vergleich in den Blick (vgl. statt vieler BAECKER 2000). Man merkt, daß bestimmte Erwartungen nicht erfüllt werden und stellt fest, daß es auch anders geht. Erst in der Konfrontation mit einer anderen Kultur, erst anläßlich von Unterschieden wird man sich überhaupt der eigenen Kultur bewußt. Dementsprechend fallen auch die Beschreibungen fremder Kulturen nicht umfassend aus; man konzentriert sich auf das, was anders ist. Das hat u.a. zur Folge, daß die Beschreibung einer Kultur immer auch die Kultur des Beobachters widerspiegelt. Angehörige verschiedener Kulturen werden auch eine dritte Kultur unterschiedlich beschreiben.

Kulturvergleiche lassen sich also immer nur aus einer bestimmten Perspektive, aus der einer bestimmten Kultur anstellen. Die Aufgabe, die Perspektive der hier folgenden Überlegungen zu verdeutlichen, wird dadurch erschwert, daß diese Verdeutlichung ebenfalls nur aus der Perspektive zu leisten ist, aus der heraus der Vergleich unternommen wird; diese Grenze läßt sich nur aus einer Beobachterperspektive überschreiten, die von anderen Prämissen ausgeht. Eine solche Beobachtung gelangte allerdings nicht zu objektiveren oder wie immer gedacht besseren Resultaten, sondern allenfalls zu anderen. Das wäre schon hilfreich genug, weil das Bild, das eine Addition von Beobachtungen aus unterschiedlichen Perspektiven bieten kann, 
umfassender ausfallen muß. Für eine solche multiperspektivische Darstellung fehlen uns jedoch zwei Voraussetzungen: Zeit/Raum und Kompetenz.

Im Bewußtsein dieser Beschränkung stellen wir die hier leitende Beobachterperspektive als die einer Teleologie ohne Ziel vor. Teleologie insofern, als einzelne Kulturen als Stationen/Erscheinungsformen eines historischen Prozesses vorausgesetzt werden, also nicht als bloß willkürlich verteilte bunte Tupfer auf dem Teppich des menschlichen Lebens. Ziellos ist diese Teleologie gleichwohl, weil keines der materialen Ziele der Geschichte unterstellt wird, wie sie der Deutsche Idealismus vorschlägt: von SCHILLERs Antrittsrede von 1789 über SCHELling, HEgEL und Richard WAGNER bis hin zu MARX und ENGELS. Unsere Formulierung verliert an Widersprüchlichkeit, wenn man - wie die von LUHMANN entwickelte Systemtheorie - Geschichte als soziale Evolution, als fortschreitende Ausdifferenzierung der Gesellschaft, zuletzt in Funktionssysteme, akzeptiert, wobei jede weitere Differenzierung Folgeprobleme hinterläßt, auf die mit weiterer Differenzierung reagiert wird. Den Übergang zu einer primär funktionalen Ausdifferenzierung der Gesellschaft und die daran anknüpfenden Prozesse werden wir in Übereinstimmung mit verbreitetem Sprachgebrauch als Modernisierung bezeichnen. Der Begriff ist analytisch zu verstehen, keineswegs wertend; modern ist nicht besser. Allerdings gehört zu den Prämissen des theoretischen Rahmens, in dem der Begriff der sozialen Evolution zentral ist, daß der Prozeß der Modernisierung langfristig unumkehrbar ist.

\subsection{Vorgehen - Thesen}

In einem ersten Schritt wird es darum gehen, eine Kategorisierung von kulturellen Unterschieden/Eigenheiten auf mehreren Abstraktionsniveaus zu begründen, so daß die Frage, wie man Erbsen auf eine Gabel bringt, nicht auf derselben Ebene verhandelt wird wie etwa die unterschiedlichen Formen des Strafvollzuges.

Im zweiten Schritt soll, in Anlehnung an HOFSTEDE, eine Ebene der kulturellen Dispositionen als die für den Kulturvergleich centrale hergeleitet und im einzelnen vorgestellt werden. 
Eingehend wird im dritten Schritt als These die Vermutung vertreten, daß kulturelle Unterschiede eher durch Unterschiede im Grad der Modernisierung, in der Gegenwart: durch den Stand der funktionalen Ausdifferenzierung der Gesellschaft begründet als durch Einzelfaktoren bis hin zum Numinosen bedingt sind. Es versteht sich von selbst, daß Modernisierung nicht von sich aus schon einen Wert darstellt; dafür ist der Preis dafür zu hoch, worauf lange vor den diversen Fundamentalismen der Gegenwart bereits NIETZSCHE aufmerksam gemacht hat (vgl. z.B. LANGE 1983).
Bedingungsfaktoren für kulturelle

Unterschiede/Merkmale

Stufe der Modernisierung

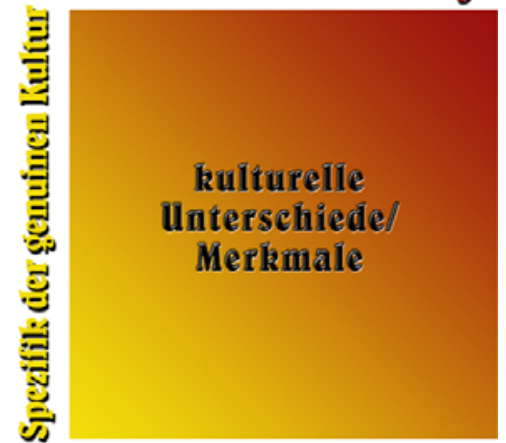

Abschließend ist exemplarisch zu überprüfen, wie weit sich die These von den unterschiedlichen Stufen der Modernisierung in bezug auf die kultur-spezifischen Dispositionen bewährt.

\section{Unterschiede Von Unterschieden}

Wenn von kulturellen Unterschieden in alltäglichen Kontexten die Rede ist, stehen zunächst Äußerlichkeiten im Vordergrund; auffällige Verhaltensformen, die den Eindruck des Fremden hinterlassen: Essen und Eßgewohnheiten, Kleidung und äußeres Erscheinungsbild, Verhalten im Gespräch, Musikpräferenzen, \&c. Gerne werden die mit der Unterschiedlichkeit von Kulturen verbundenen Probleme auf diese Ebene reduziert, für die Norbert BolTZ die Bezeichnung BoutiqueMultikulturalismus findet (2002: 27). Wir wählen hierfür die Sammelbezeichnung offenes Verhalten.

Eine nähere Beschäftigung mit Beobachtungen dieser Art führt zu dem Auffinden von Regeln, die den irritierenden Verhaltensformen zugrundeliegen und die die Verhaltenserwartungen innerhalb einer Kultur begründen: Man sieht im Aufenthaltsraum, daß nur wenige in den Sesseln rechts und links vom Kamin Platz nehmen, stellt fest, daß dies ausschließlich die Abteilungsleiter tun, und gelangt zu der Regel: Diese 
Plätze sind für Abteilungsleiter, auch wenn sich gerade niemand von ihnen im Raum befindet, reserviert. Im Zweifelsfall kann man nachfragen oder wird freundlich-dezent darauf aufmerksam gemacht. Solche Regeln kann man etwa in entsprechenden Vorbereitungsveranstaltungen für einen Auslandsaufenthalt lernen. In vielen Fällen lassen sich solche Regeln rational nicht weiter begründen (das ist eben so!), in anderen können sie aus der Tradition hergeleitet werden (vgl. LÜBBE 1973); in wieder anderen können sie rekonstruiert werden (vgl. ELIAS 1969). Die Vertrautheit mit Regeln dieser Art ermöglicht es nicht nur, das eigene Verhalten auf die Erwartungen von Angehörigen einer anderen Kultur abzustellen; sie ist zugleich die Voraussetzung dafür, das Verhalten von Angehörigen einer anderen Kultur angemessen aufzunehmen.

So hilfreich wie unverzichtbar die Vertrautheit mit dieser Art Regeln ist, es bleibt eine Reihe von Defiziten, u.a.:

1. Regeln haben eine ...wenn, dann ...-Struktur; ihre erfolgreiche Anwendung setzt übereinstimmende Situationsdefinitionen durch die Partner voraus.

2. Nicht alle Situationen werden durch Regeln abgedeckt; es gibt immer Ausnahmen.

3. Niemand kann alle Regeln kennen.

Ein weiterer Aspekt: Regeln der genannten Art dürften in einer Kultur kaum isoliert nebeneinanderstehen; es ist mit systematischen Zusammenhängen zu rechnen.

$\mathrm{Zu}$ fragen ist mithin nach der Basis für Orientierungen in Situationen, für die keine eindeutigen Regeln zur Verfügung stehen. Im alltäglichen Verkehr sind mit Blick auf andere Menschen jene Verhaltenserwartungen unverzichtbar, die sich auf die Reaktionen anderer in Situationen beziehen, die nicht mehr durch das Befolgen von gruppenverbindlichen Regeln fortsetzbar sind. Man kennt den anderen als gutmütig oder hinterhältig, als zuverlässig oder schlampig, und kann sich darauf einrichten. Aufgrund solcher Erwartungen kann man abschätzen wenngleich nicht wissen -, wie sich der andere in einer bestimmten zukünftigen Situation verhalten wird; unter Umständen wird der andere auf dieser Grundlage sogar kalkulierbar. Ein markantes Beispiel hierfür ist die Veröffentlichung der redigierten Fassung eines Telegramms vom 
König von Preußen durch BISMARCK (1898: II, 91), bekannt als Emser Depesche, die NAPOLEON III. mit der Kriegserklärung an Preußen reagieren ließ.

Es gehört $\mathrm{zu}$ den Begleiterscheinungen von Begegnungen mit Angehörigen anderer Kulturen - und nicht allein von Landeskulturen -, $\mathrm{da} ß$ in Analogie $\mathrm{zu}$ interpersonalen Erfahrungen entsprechende Erwartungen und Zuschreibungen in bezug auf soziale Gruppen gebildet bzw. vorgenommen werden.

Wir wollen solche erwartungsbildenden Eigenschaften als Verhaltensdispositionen bezeichnen; dieser Ausdruck soll den üblichen, im einzelnen jedoch unbestimmten und mit den unterschiedlichsten philosophi-schen und psychologischen Implikationen belasteten Terminus Charakter/Nationalcharakter ablösen (vgl. u.a. HOFSTÄTTER 1957; THOMAS 1993). Wesentliche Anregungen für die Präzisierung dieser Ebene der (kulturspezifischen) Verhalternsdispositionen beziehen wir aus den Arbeiten von Geert Hofstede (1984; 1991a; 1991b). Kulturspezifische Dispositionen sind danach die in einer Kultur eingelebten, im Laufe der Sozialisation erworbenen Grundlagen des Entscheidens und kommunikativen Verhaltens. Mit dem Begriff $<$ Verhaltensdisposition> wird der des mental program von HOFSTEDE (1984) aufgegriffen und fortgesetzt, der für ihn die Unterschiede erklärbar macht:

Every person carries within him or herself patterns of thinking, feeling, and potential acting which were learned throughout their lifetime. Much of it has been acquired in early childhood, because at that time a person is most susceptible to learning and assimilating. [...]

Using the analogy of the way in which computers are programmed, this book will call such patterns of thinking, feeling, and acting mental programs, or, as the sub-title goes: 'software of the mind'. This does not mean, of course, that people are programmed the way computers are. A person's behavior is only partially predetermined by her or his mental programs: [...] The 'software of the mind' [...] only indicates what reactions are likely and understandable, given one's past. (1991a: 4)

Die Einschränkung am Ende des Zitates verdient besondere Beachtung: es geht um keinen Automatismus, wie ihn andere Autoren 
und Theorien unterstellen, die die Programmierbarkeit von Menschen feilbieten, wie etwa die Lehre vom neuro-linguistischen Programmieren (vgl. statt vieler KRUSCHE: Der Frosch auf der Butter. 1992).

Mitgliedern einzelner Kulturen werden jeweils spezifische Verhaltensdispositionen im Sinne von Entscheidungs- und Handlungsgrundlagen zugeschrieben, sowohl als Fremd- wie als Selbstbilder. Als Beispiel mag hier die von Russen erwartete Entscheidungsschwäche und Handlungszurückhaltung dienen, wie sie die Titelfigur von GONTSCHAROWs Roman Oblomov verkörpert. Dabei handelt es sich nicht allein um ein Vorurteil aus Außensicht, sondern ebenso - wenn nicht sogar noch ausgeprägter - um ein unter der Bezeichnung oblomovwina vertrautes und gerne zitiertes Element des Selbstverständnisses.

Kulturspezifische Dispositionen wie auch Verhaltensregeln lassen sich mehr oder weniger stringent aus Hintergrundphänomenen wie Tradition, Religion, Wertorientierungen, \&c., einem geistigen Hintergrund, herleiten, zwischen denen in einer Weise, die hier nicht weiter zu verfolgen ist, Interdependenzen bestehen. Für die Frage nach kulturellen Unterschieden gewinnen sie dann Bedeutung, wenn sie auf den anderen Ebenen manifest werden.

Die Beschäftigung mit kulturellen Unterschieden setzt die Vertrautheit mit dieser und den Bezug auf diese Ebene der Hintergrundphänomene voraus, macht diese jedoch nicht zu ihrem Gegenstand; dadurch läßt sie sich etwa von der vergleichenden Religionswissenschaft abgrenzen.

Die Ebene der kulturspezifischen Dispositionen ist für unsere Fragestellung die Schlüsselebene. Anders als in Fällen offenen Verhaltens, wo sich aufgrund von erlernten bzw. erlernbaren Regeln Erwartungen in bezug auf Handeln/Verhalten der anderen bilden lassen, stellen kulturspezifische Dispositionen Handlungsorientierungen für beliebige Situationen zur Verfügung und damit auch den Rahmen für Erwartungen in Situationen, für die es keine festen Regeln gibt. Wer damit vertraut ist, daß in der Kultur des Partners z.B. hierarchisch begründete Rücksichten eine größere Bedeutung haben als in der eigenen, wird dies bei Verhand-lungen berücksichtigen, ohne daß dies eigens thematisiert wird - wozu es in vielen Fällen auch gar nicht kommen kann. 
Anders als das, was wir als den geistigen Hintergrund bezeichnen, sind jedoch kulturspezifische Dispositionen noch so konkret, daß sie als Handlungsorientierungen formulierbar sind. Sie enthalten, um einen Begriff aus der Rhetorik zu verwenden, die Topoi des Verhaltens in einer Kultur.

Der Vorschlag, kulturelle Unterschiede auf den hier vorgestellten vier Ebenen zu unterscheiden, ist pragmatisch bestimmt; es handelt sich um Stufen aufsteigender Abstraktion von Beobachtungen, die auch mit anderem Ergebnis festgelegt werden könnten und sich keinesfalls aus der Natur der Sache ergeben; zusammenfassend:

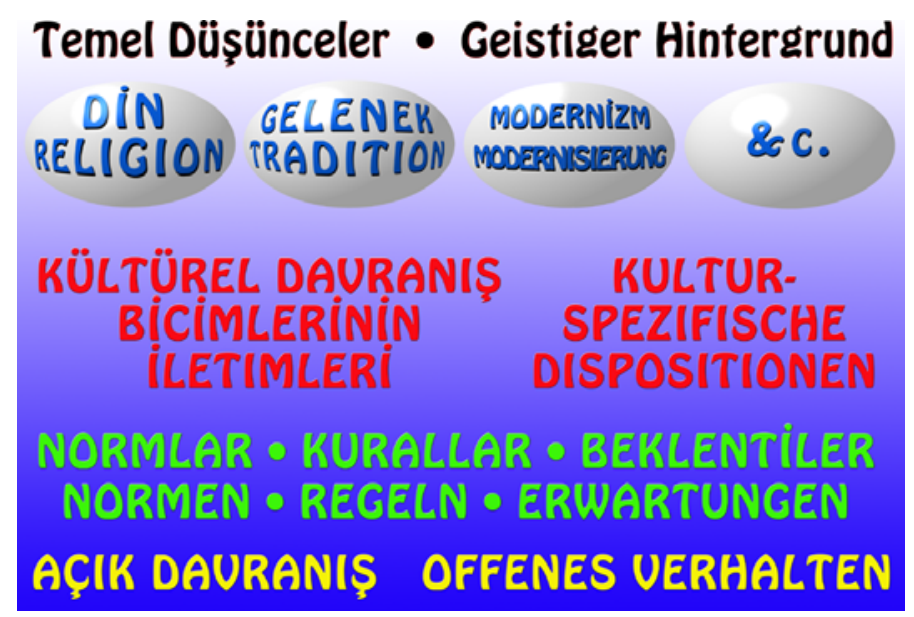

Diese Anordnung ist auch methodisch von Bedeutung: Sie ermöglicht nicht nur eine erste systematische Ordnung der Beobachtungen kultureller Unterschiede; sie bietet auch den Rahmen für die Hypothese, daß zwischen Beobachtungen auf unterschiedlichen Ebenen Bedingungszusammenhänge bestehen. Wir werden dies mit Bezug auf die Modernisierung (s.u.) ausführlicher exemplarisch darstellen und dabei geichzeitig bemüht sein, der Gefahr von Systemzwängen zu erliegen.

\section{Kulturspezifische Dispositionen}

Zur Systematisierung dieser Grunddispositionen bietet sich das sog. Organon-Modell der Kommunikation von BÜHLER (1934); danach erfüllt jedes in Kommunikation verwendete Zeichen gleichzeitig - wenngleich im Einzelfall in unterschiedlich starker Ausprägung - drei Funktionen: 


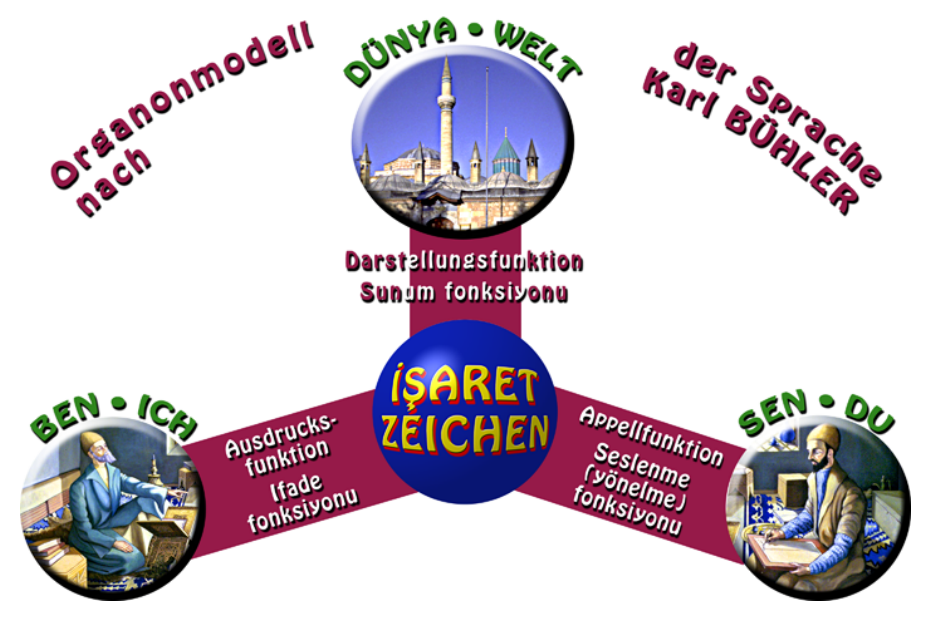

1. AuSDRUCKSfunKtion. Der Sprecher bringt sich selbst zum Ausdruck, stellt sich selber dar.

2. Appellfunktion. Der Sprecher nimmt Beziehungen zum Partner auf und gestaltet diese.

3. DarstellungsfunkTiOn. Der Sprecher bezieht sich auf die Wirklichkeit, die Welt.

Diese Funktionstypen haben ihre Entsprechung in der grammatischen Struktur der Personalpronomina - zumindest in den indogermanischen Sprachen und dem Flexionssystem der Verben; im Deutschen: ich, $d u$, er/sie/es.

In der Erweiterung des BÜHLERschen Modells durch JAKOBSON (1960) werden - dies ist für unsere Überlegungen hilfreich - bei der Appellfunktion BÜHLERs zwei Funktionen unterschieden: (1) die conative function, die Beziehungen zum Partner herstellt und aufrecht erhält, und (2) die phatic function, die der Sicherung des Kommunikationskontaktes dient.

Aus der formalen Funktionsbestimmung BÜHLERs lassen sich spezifischere Funktionen der Kommunikation für Menschen herleiten: 


\section{Allgemeine Funktionen Der Kommunikation Für Den Menschen}

İnsanlar İçin İletişsimin Genel Fonksiyonlari

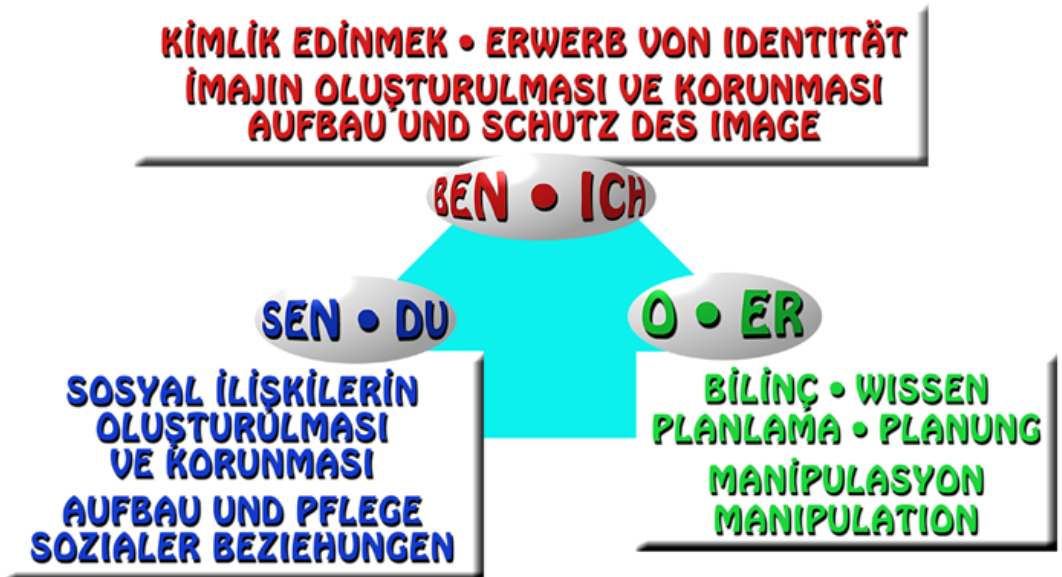

Kulturspezifische Dispositionen sind die in einer Kultur eingelebten, im Laufe der Sozialisation erworbenen Grundlagen des Entscheidens und kommunikativen Verhaltens. Ausgangspunkt für unseren Versuch einer Systematisierung sind die von HOFSTEDE vorgestellten mental programs; auf der Grundlage anderer Forschungen sowie eigener Beobachtungen wurden diese teilweise modifiziert und ergänzt. Bei einer solchen empirischen Basis ist damit zu rechnen, daß die Liste ergänzbar ist.

Die genannten Dispositionen lassen sich zwar unterscheiden, sind jedoch nicht voneinander isoliert; es bestehen Abhängigkeits- und Bedingungsverhältnisse, ohne daß es allerdings möglich oder auch nur sinnvoll ist, die einzelnen Dispositionen in ein unversales System zu zwängen.

Da die Zusammenstellung der kulturspezifischen Dispositionen dazu dienen soll, kulturelle Unterschiede zu fassen, werden sie als Differenzen formuliert; wo dies nicht explizit geschieht (z.B. 1.3 $<$ Entscheidungsfreudigkeit>), lautet die Differenz ja/nein, hoch/niedrig oder entsprechend. Der übliche Fall ist jedoch nicht der der Alternative, sondern die Angabe von Endpunkten einer Skala, auf der sich die Angehörigen einzelner Kulturen lokalisieren lassen (müssen). 
Im folgenden stellen wir zunächst eine unkommentierte Liste solcher Dispositionen vor, die sich bei unserer bisherigen Beschäftigung mit dem Themenkomplex ergeben haben:

\section{KÜLTÜREL DAVRANIŞ BİÇIMLERİNIN İLETIMMLERİ}

$$
\text { KULTURSPEZIFISCHE DISPOSITIONEN }
$$

İfade fonksiyonu («BEN») • Ausdrucksfunktion («ICH»)

\begin{tabular}{|c|c|c|}
\hline 1.1 & Birey - Kollektif & Individuum - Kollektiv \\
\hline 1.2 & Özel-Aleni & Privat - Öffentlich \\
\hline 1.3 & Karar verme yetisi & Entscheidungsfreudigkeit \\
\hline 1.4 & İçe dönüklük - Dışa dönüklük & Introvertiert - Extrovertiert \\
\hline 1.5 & Öz yansıma & Selbstreflexivität \\
\hline \multicolumn{3}{|c|}{ Seslenme (yönelme) fonksiyonu («SEN») • Appellfunktion («DU») } \\
\hline 2.1 & Yakınlık - Uzaklık & Nähe - Distanz \\
\hline 2.2 & Egemenlik - Benimsenmiş Roller & $\begin{array}{l}\text { Egalitär - Ausgeprägte } \\
\text { rollenspez. Beziehungen }\end{array}$ \\
\hline 2.3 & Tasdiklemek (sosyal ve sembolik) & $\begin{array}{l}\text { Symbolische soziale } \\
\text { Anerkennung }\end{array}$ \\
\hline 2.4 & $\begin{array}{r}\text { Sosyal farklılıklar / } \\
\text { Yönlendirmeler }\end{array}$ & $\begin{array}{l}\text { Soziale Differenzierung / } \\
\text { Orientierung }\end{array}$ \\
\hline 2.5 & $\begin{array}{r}\text { Anlaşmazlıklara açık olma/ } \\
\text { Anlaşmazlıklarda davranış } \\
\text { biçimleri }\end{array}$ & $\begin{array}{l}\text { Konfliktbereitschaft / } \\
\text { Konfliktverhalten }\end{array}$ \\
\hline 2.6 & $\begin{array}{r}\text { Sosyal davranış / } \\
\text { Sosyal sorumluluk }\end{array}$ & $\begin{array}{l}\text { Soziales Verhalten / } \\
\text { Soziale Verantwortung }\end{array}$ \\
\hline 2.7 & İlişkilerde İletişim üslubu & $\begin{array}{l}\text { Stil der Kommunikation von } \\
\text { Beziehungen }\end{array}$ \\
\hline 2.8 & Organizenin değeri ve anlayışı & $\begin{array}{l}\text { Organisationsgrad und - } \\
\text { verständnis }\end{array}$ \\
\hline 2.9 & formal / formal olmayan & $\begin{array}{l}\text { Norm und Aus-Zeit } \\
\text { formell/informell }\end{array}$ \\
\hline \multicolumn{3}{|c|}{ Sunum fonksiyonu $(« O ») \cdot$ Darstellungsfunktion $(« E R »)$} \\
\hline 3.1 & Yaşanan / ölçülen zaman & erlebte / gemessene Zeit \\
\hline 3.2 & $\begin{array}{r}\text { Rasyonallik / } \\
\text { Mit'lere göre dünya anlayışı }\end{array}$ & $\begin{array}{l}\text { Rationales / mythisches } \\
\text { Weltverständnis }\end{array}$ \\
\hline 3.3 & Bu dünya - Öteki dünya anlayışı & Diesseits - Jenseitsorientierung \\
\hline 3.4 & Değişimlere açık olmak & $\begin{array}{l}\text { Veränderungsvemögen } \\
\text { /-bereitschaft }\end{array}$ \\
\hline
\end{tabular}


Über weite Strecken ist dieser Katalog noch als Arbeitsprogramm zu verstehen, dessen einzelne Punkte weiter zu konkretisieren bleiben. Zur Verdeutlichung wird je eine der genannten Dispositionen aus den drei Funktionsbereichen exemplarisch vorgestellt.

\subsection{Ausdrucksfunktion:}

\section{$<1.2$ Privat/Öffentlich $>$}

In der Türkei muß man eher als in Deutschland darauf eingerichtet sein, im Falle einer flüchtigen Bekanntschaft, wie sie sich etwa bei einer längeren Reise ergibt, auf private Dinge wie Gesundheit/Krankheit, Familie, Einkommen und Vermögensverhältnisse, \&c. angesprochen zu werden; das schließt natürlich ein, daß auch der Partner bereit ist, seine eigene Situation in diesen Punkten darzulegen. Wir nehmen dies als ein Indiz dafür, daß sich Kulturen darin unterscheiden, daß die Grenze zwischen privat und öffentlich unter-schiedlich verläuft.

\subsection{Appellfunktion:}

\section{$<2.1$ Nähe/Distanz $>$}

Wohl in jeder Kultur findet sich ein kompliziertes System von Formen der Anrede. Läßt man die Verwendung von Titeln einmal außer acht, so kann man in Deutschland zwei für die Anrede verbindliche Unterscheidungen finden: $<d u / S i e>$ und $<$ Vorname/Nachname $>$, die in Standardkombinationen unter Angehörigen der deutschen Kultur verwendet werden: 


\begin{tabular}{|c|c|c|}
\hline & VORNAME & NACHNAME \\
\hline$D U$ & $\begin{array}{l}\text { I. } \\
\text { wechselseitig: } \\
\text { 1. } \text { STANDARD: innerhalb der } \\
\text { Familie } \\
\text { 2. } \text { STANDARD: unter guten } \\
\text { Bekannten (ggf. nach förmlicher } \\
\text { Übereinkunft) } \\
\text { 3. STANDARD: unter Jugendlichen } \\
\text { 4. HÄUFIG: unter Arbeitskollegen } \\
\text { einseitig: } \\
\text { 5. STANDARD: gegenüber Kindern } \\
\text { 6. HÄUFIG: gegenüber Untergebenen } \\
\text { 7. } \text { GELEGENTLICH: gegenüber } \\
\text { D. Dienstpersonal } \\
\text { HÄUFIG: (geringschätzig) bei } \\
\text { Annahme von erheblicher sozialer } \\
\text { Distanz }\end{array}$ & $\begin{array}{l}\text { III. } \\
\text { wechselseitig, unter Fortlassen von } \\
\text { Herr/Frau: } \\
\text { 1. STANDARD: unter Schülern einseitig: } \\
\text { 2. HÄUFIG:in Abhängigkeitsverhältnissen } \\
\text { (z.B. Lehrer/Schüler) wechselseitig, in } \\
\text { Kombination mit Herr/Frau: } \\
\text { GELEGENTLICH REGIONAL: } \\
\text { 3. am Arbeitsplatz } \\
\text { 4. in verschiedenen sektoralen } \\
\text { Zusammenhängen }\end{array}$ \\
\hline SIE & $\begin{array}{l}\text { II. } \\
\text { wechselseitig: } \\
\text { GELEGENTLICH REGIONAL: } \\
\text { 1. am Arbeitsplatz } \\
\text { 2. in verschiedenen sektoralen } \\
\text { Zusammenhängen }\end{array}$ & $\begin{array}{l}\text { IV. } \\
\text { wechselseitig: } \\
\text { 1. STANDARD: unter Personen, die sich } \\
\text { gar nicht oder nur flüchtig oder } \\
\text { nur aus dem Arbeitszusammenhang } \\
\text { kennen }\end{array}$ \\
\hline
\end{tabular}

Die leitende Unterscheidung $<d u+$ Vorname $>/<$ Sie + Nachname $>$ (Varianten I und IV) löst seit dem 19. Jahrhundert eine Vielzahl von Anderenformen ab und führt zu einer Vereinheitlichung. Hintergrund für diesen Wandel ist ein Wandel in den Kriterien sozialer Differenzierung: die primär hierarchische Differenzierung der Gesellschaft geht über in eine letzlich primär funktionale, die die Gleichheit aller Menschen zur Voraussetzung hat und in der vor allem - mittels der Unterscheidung $<$ du/Sie $>$ - die Unterscheidung < soziale Nähe/Ferne $>$ herausgehoben ist (vgl. SANDER 1998). Dem widerspricht weder, daß auch in früheren Zeiten <soziale Nähe/Ferne> eine Rolle spielte und die Differenz in Anredeformen ihren Ausdruck fand; noch, daß auch die gegenwärtige Praxis hierarchische Momente enthält. Wer einer ihm fremden Person die 
Anredeform Sie verweigert, verweigert ihr damit auch den $\mathrm{zu}$ erwartenden Respekt.

Anders liegen die Dinge in der Türkei:

\begin{tabular}{|c|c|c|c|}
\cline { 2 - 4 } \multicolumn{1}{c|}{} & VORNAME & $\begin{array}{r}\text { VORNAME + -BEY, -HANIM, } \\
\& \mathrm{C} .\end{array}$ & NACHNAME \\
\hline SEN & $\begin{array}{c}\text { korrekt unter } \\
\text { Gleichaltrigen, } \\
\text { Gleichgestellten } \bullet \text { sehr } \\
\text { vertraut }\end{array}$ & $\begin{array}{c}\text { korrekt unter } \\
\text { Gleichgestellten } \bullet \text { vertraut }\end{array}$ &. \\
\hline$S<Z$ &.$/$ & korrekt, mit Respekt & offiziell, distanziert \\
\hline
\end{tabular}

Bei so unterschiedlichen Ausgangslagen sind Mißverständnisse und Irritationen vorhersehbar, da Intentionen und Verstehen stark auseinandergehen können:

\begin{tabular}{|c|c|c|}
\cline { 2 - 3 } \multicolumn{1}{c|}{} & $\begin{array}{c}\text { AUS DEUTSCHER SICHT } \\
\text { INTENDIERT / ZU VERSTEHEN }\end{array}$ & $\begin{array}{c}\text { AUS TÜRKISCHER SICHT ZU } \\
\text { VERSTEHEN }\end{array}$ \\
\hline DU + VORNAME & geringschätzig bis feindselig & freundlich, wohlwollend \\
\hline SIE + NACHNAME & korrekt, mit Respekt & unfreundlich bis feindselig \\
\hline
\end{tabular}

Nicht selten war im Westdeutschland der 60er Jahre zu beobachten, daß viele Menschen aus der Türkei, die zur Erwerbstätigkeit nach Deutschland gekommen waren, diejenigen Vorgesetzten und Behördenvertreter eher als angenehm empfanden, die vom Verhalten her, mit der Anrede $d u+$ Vorname, eher distanziert oder herablassend auftraten, während korrektes Verhalten (Sie + Nachname) oft als unangenehm erlebt wurde, was dann unzutreffende Erwartungen zur Folge hatte. 


\title{
3.3. Darstellungsfunktion:
}

\section{<3.2 Rationales/Mythisches Weltverständnis>}

Rationales Weltverständnis geht von der prinzipiellen Erklärbarkeit und Verstehbarkeit der Welt aus. Die Zeichen (Worte oder Bilder), mit denen kommuniziert wird, werden unterschieden gesehen von den Gegenständen oder Sachverhalten, zu deren Bezeichnung sie dienen; sie sind - wie die Sprache überhaupt - Instrumente zum Zwecke der Verständigung; insgesamt steht für rationales Denken ein angebbarer Zweck als Handlungsziel im Vordergund. Die Zwecke werden durch die einzelnen Funktionssysteme der Gesellschaft verbindlich gemacht: als Präferenzwerte von deren Leitdifferenzen (vgl. LUHMANN 1986); so z.B.:

\author{
Wirtschaft Gewinn \\ Politik Macht \\ Wissenschaft (methodisch abgesicherte) Wahrheit \\ Bildung Karriere
}

Die Verfolgung dieser Zwecke wird primär kontrolliert durch die Eigenlogik des jeweiligen Systems wie durch die Resonanz der übrigen Systeme, allen voran der Rechtsordnung; nicht jedoch von beziehungsbedingten Rücksichten, solchen auf die Tradition oder die Religion.

\section{Tradition Oder Moderne}

Nach verbreiteter Auffassung sind die Merkmale der einzelnen Kulturen und - damit verbunden - die Unterschiede zwischen ihnen - als gegeben hinzunehmen. Die Merkmale/Unterschiede lassen sich zwar historisch herleiten und erklären, etwa aus früheren Lebensgewohnheiten oder religiösen und mythischen Vorstellungen; aber damit wird das Problem nur vorverlegt; man nimmt dann die ursprünglichen Merkmale/ Unterschiede als gegeben hin.

Vergleichende Ethnologie gelangt - bei erheblichen Unterschieden im einzelnen - über Beobachtungen zur Ermittlung übergreifender menschlicher/gesellschaftlicher Grundfunktionen, für deren Erfüllung sich in den einzelnen Kulturen - systemtheoretisch formuliert unterschiedliche funktionale Äquivalente finden lassen (z.B. FRAZER 
1922). In bezug auf Rituale etwa war VAN GENNEP aufgrund umfangreicher Feldforschungen schon früh (1909) zu dem Schluß gekommen, daß in menschlichen Gesellschaften das Überschreiten von Grenzen zeitlicher, räumlicher, biographischer und sozialer Art besonders markiert werde; daß Rituale, die in allen Gesellschaften zu finden seien, diesem Zweck dienten; und daß die Formen der Rituale aus der Stuktur des Überganges (Abschied vom Alten, eigentlicher Wandel, Ankunft im Neuen) zu erklären seien; die konkreten Ausprägungen in den einzelnen Kulturen bleiben jedoch auch damit unerklärt. Strukturalistische Erklärungen, etwa von LÉVI-STRAUSS (1958) oder PIKE (1967), stellen theoretisch begründete Ansätze für eine Systematik kultureller Merkmale/Unterschiede vor, ohne zu versuchen, die beobachtbaren Unterschiede herzuleiten. Wir werden im folgenden solche Merkmale/Unterschiede, die sich einer übergreifenden Systematisierung entziehen und die als nicht weiter hintergehbare Merkmale/Unterschiede hingenommen werden, als genuine Merkmale/Unterschiede bezeichnen.

Eine gewisse Vertrautheit mit unterschiedlichen Kulturen führt sehr bald zu der Beobachtung, daß die Unterschiede zwischen den Bewohnern von Metropolen verschiedener Kulturen deutlich geringer sind als zwischen den Bewohnern ländlicher Regionen. Begründet ist die Vermutung u.a. damit, daß eine Reihe von Unterschieden, die als genuin kulturelle wahrgenommen werden, günstiger mit unterschiedlichen Stufen im Prozeß der Modernisierung in dem oben eingeführten Sinne in Verbindung zu bringen sind. Gestützt wird diese Vermutung durch die Tatsache, daß Großstädte/Metropolen in allen Kulturen die Zentren der Modernisierung sind. Gelegentlich entsteht sogar der Eindruck, daß die Unterschiede zwischen Bewohnern von Metropolen unterschiedlicher Landeskulturen (z.B. denen von «stanbul und Berlin) geringer sind als die Unterschiede zwischen denen der Metropolen und denen der jeweiligen Provinz.

Aus dieser Vermutung ergibt sich eine methodische Konsequenz: Ausgangspunkt für die weitere Forschung ist die Prämisse, daß sich landeskulturelle Merkmale/Unterschiede auf Unterschiede im Grad der Modernisierung zurückführen lassen; $<$ modern/nicht modern $>$ ist damit die Leitdifferenz für die Beobachtung. 
Diese methodische Prämisse ist keine Forschungshypothese, die es zu überprüfen gilt. Sie stellt lediglich den Untersuchungsrahmen dar und rechnet damit, daß sich Merkmale/Unterschiede finden werden, die nicht mit dem Stand der Modernisierung in Verbindung gebracht werden können, die bei diesem Vorgehen als genuine Merkmale/Unterschiede einzustufen, die z.B. nur aus der Tradition zu begründen sind.

Gleichwohl ist die Leitdifferenz $<$ modern/nicht modern $>$ nicht willkürlich gewählt:

1. Sie ergibt sich aus der Beobachtung von Merkmalen/Unterschieden und deren Rezeption in unterschiedlichen Kulturen.

2. $\mathrm{Zu}$ den Annahmen dieses Ansatzes gehört, daß die soziale Evolution, der Prozeß der Modernisierung global und unumkehrbar ist; dies ist unter einem anspruchsvollen Begriff von Globalisierung mitgedacht. Sofern sich kulturelle Merkmale/Unterschiede aus dem Modernisierungsproze $\beta$ herleiten lassen, kann eine solche Einsicht als hilfreiche Entscheidungsgrundlage in einer Vielzahl von Situationen dienen.

3. Kulturelle Merkmale/Unterschiede, die in einem erkennbaren Zusammenhang mit der Modernisierung stehen, werden - so die mit dem Konzept der Globalisierung verknüpfte Folgerung - im weiteren Verlauf der Modernisierung in den Hintergrund treten; gelegentlich ist von einer Globalkultur die Rede. Eine Analyse von kulturellen Merkmalen/Unterschieden unter dem Aspekt der Modernisierung kann in zwei Punkten zur Klärung beitragen:

a. bei der Frage nach der Rolle und den Chancen von genuinen Merkmalen/Unterschieden im Prozeß der Modernisierung; dies scheint vor allem für Kulturen von Bedeutung $\mathrm{zu}$ sein, die in einem beschleunigten Modernisierungsproze $\beta$ ihre Identität bedroht sehen;

b. angesichts der Zwangsläufigkeit, die vielfach für die Entwicklung hin zu einer Globalkultur reklamiert wird; und der verbreiteten Hilflosigkeit, mit der solchen Thesen Szenarien entgegengehalten werden, die mit kaum mehr als der Freude am Pittoresquen und dem 
Wunsch nach etwas begründet sind, was an einen Kulturzoo denken läßt, in dem vor allem Folklore für Kultur gehalten wird.

Basis der methodischen Prämisse unserer Argumentation ist die Position der Moderne; andere Positionen könnten zu anderen Ergebnissen führen. Diese Basis schränkt die Möglichkeiten der Methode auch ein: sie ist kaum tauglich für den Vergleich von Kulturen, die sich bei erheblichen Unterschieden auf derselben Stufe der Modernisierung befinden.

\subsection{Zum Begriff Der Moderne}

Mit dem Begriff des Fortschritts als Ziel- und Positionsangabe auf dem Weg der gesellschaftlichen Entwicklung impliziert der Begriff der Moderne eine Wertung. Man kann die These von der Evolution der Gesellschaft jedoch ebenso akzeptieren und zu einer gegenteiligen Bewertung gelangen; der dem entsprechende Ausdruck lautet Dekadenz. Fragen und Probleme dieser Art liegen außerhalb des hier verfolgten Interesses, das sich auf die Entdeckung systematischer Zusammenhänge richtet.

Das Konzept der Evolution enthält die Annahme von deren Unumkehrbarkeit. Die Prämisse, daß kulturelle Unterschiede primär als systembedingte zu sehen sind, schließt mithin eines weitestgehend aus: die Realisierung sozialromantischer Hoffnungen, wie sie Reisende in mittägliche Regionen gerne aus dem Urlaub zurückbringen: die moderne Lebenswelt mit sympathischen Versatzstücken vormoderner Lebensformen anzureichern. Gleichermaßen abwegig ist das Spiegelbild, wo die Ausstattung mit Accessoires der Moderne mit Modernisierung verwechselt wird.

Mit dem Begriff der Modernisierung folgen wir der Bestimmung der von LUHMANN entwickelten Systemtheorie; danach ist im Konzept der sozialen Evolution der Prozeß der Modernisierung als eine Abfolge von Typen sozialer Differenzierung zu sehen. Die ursprüngliche Form ist die der horizontalen Differenzierung, die Gliederung der Gesellschaft auf der Grundlage verwandtschaftlicher, also biologisch begründeter Bindungen. Diese wird abgelöst durch eine hierarchische Differenzierung, in der sich Unterschiede auf einer Rangskala von Schichten oder Klassen 
herausbilden; der Übergang zu diesem Typ der Differenzierung steigert die Leistungsfähigkeit einer Gesellschaft, insbesondere in bezug auf äußere und innere Sicherheit, durch die Herausbildung eines eigenen Bereichs des Politischen; der Leviathan von HOBBES (1651) kann hier als ein Markstein gesehen werden. Die Industrialisierung ist begleitet von einem Wechsel hin $\mathrm{zu}$ einer Differenzierung in gesellschaftliche Funktionsssysteme; d.h. die Industrialisierung setzt eine funktionale Differenzierung ebenso voraus wie diese die Industrialisierung. Konkurrierende Begriffe von Modernisierung sind in dieses Differenzierungskonzept integrierbar: solche, die den technischen Fortschritt, Demokratisierung, Rechtssicherheit und bürgerliche Freiheiten oder Durchsetzung der Geldwirtschaft zum Auzsgangspunkt nehmen.

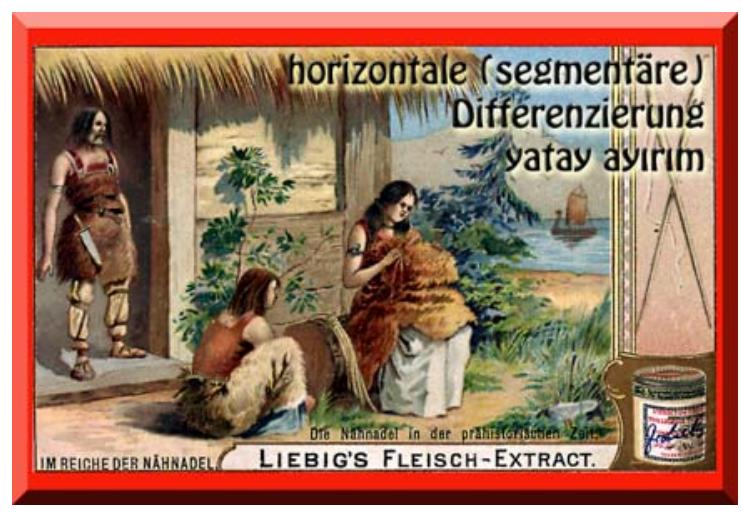

In segmentären Gesellschaften erfolgt die Inklusion eindeutig über Zuordnung zu bestimmten Segmenten der Gesellschaft, und zwar auf der konkreten und lebenswichtigen Ebene von Kleinsteinheiten der Wohnund Lebensgemeinschaften. Die Zuordnung zu größeren Einheiten (Stämmen, clans) regelt sich von da her und kann bereits hochgradig fiktive Beschreibungen benutzen - vor allem die Symbolik gemeinsamer Abstammung. Die Inklusion folgt festen Regeln (zum Beispiel Heiratsregeln, Aufnahmeregeln) [...]. 


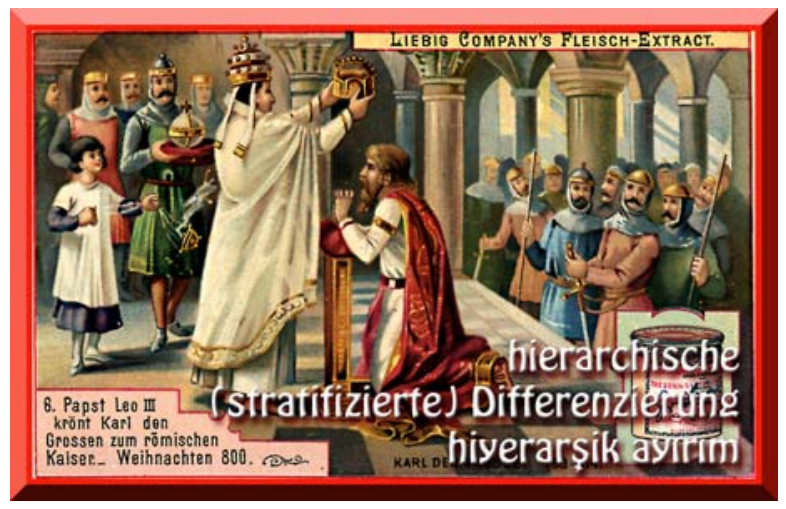

In stratifizierten Gesellschaften folgt die Inklusion ebenfalls dem Differenzierungsprinzip. Man gehört zu einer Gesellschaft auf Grund der Zugehörigkeit zu einer Kaste, einem Stand, einer bestimmten Schicht, die über Inklusion/Exklusion, und das heißt vor allem: über Endogamie, geschlossen wird. Wie in segmentären Gesellschaften kann man nur einem und nicht mehreren Teilsystemen angehören. Individualität wird durch Zuweisung eines sozialen Status erworben. Dafür hält die Gesellschaft jetzt jedoch mehrere, nicht nur gleiche, sondern ungleiche Möglichkeiten bereit.

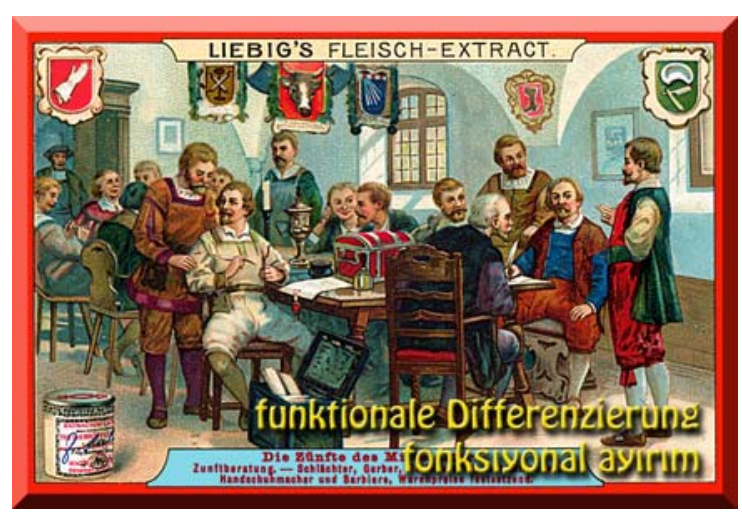

Aus strukturellen Gründen muß die moderne, funktional differenzierte Gesellschaft auf eine gesellschafts-einheitliche Regelung von Inklusion verzichten. Sie überläßt diese Frage ihren Funktionssystemen. 


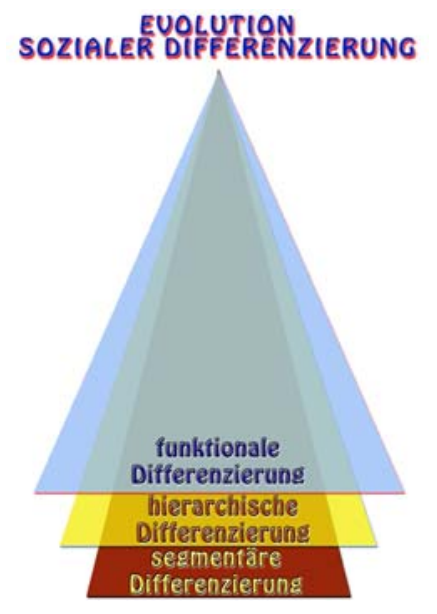

Das Verständnis von Modernisierung als soziale Evolution, wo verschiedene Typen sozialer Differenzierung aufeinander folgen, fordert keine radikale Ablösung des einen durch den anderen - gleichsam über Nacht. Vielmehr geht es um den jeweils primären, dominierenden Typ sozialer Differenzierung, wobei die späteren Formen sich bereits in den früheren herausbilden und die älteren fortbestehen; auch in einer funktional differenzierten Gesellschaft hat die Familie noch eine Bedeutung und sind hierarchische Ordnungen noch nicht verschwunden. Bei kulturellen Merkmalen/Unterschieden wird es also eher um unterschiedliche Gewichtungen gehen.

\section{Kulturelle Unterschiede Als Modernsierungsunterschiede}

Die bisherigen Überlegungen führen zu zwei methodischen Prämissen:

1. Landeskulturelle Unterschiede lassen sich auf mehreren Ebenen einer Skala <konkret/abstrakt $>$ anordnen. Aus heuristischen Gründen gehen wir von vier Ebenen aus, die jedoch keinesfalls notwendig aus der Natur der Sache hergeleitet werden können:

Geistiger Hintergrund

Kulturspezifische Dispositionen

Normen, Regeln, Erwartungen

Offenes Verhalten 
Zwischen den einzelnen Merkmalen/Unterschieden bestehen Abhängigkeitsverhältnisse; d.h. eine be stimmte Form offenen Verhaltens folgt bestimmten Regeln, die in einer kulturspezifischen Disposition begründet sind; diese wiederum ist von einer bestimmten Ausprägung des geistigen Hintergrundes abhängig. Die Ebene der kulturspezifischen Dispositionen ist für unsere Fragestellung die Schlüsselebene.

2. Kulturelle Merkmale/Unterschiede resultieren aus unterschiedlichen Etappen im Prozeß der Modernisierung. Wo sich eine dementsprechende Zuordnung nicht finden läßt, ist bis auf weiteres davon auszugehen, daß es sich um genuine Merkmale/Unterschiede handelt.

Im folgenden sollen beide Perspektiven exemplarisch miteinander verbunden werden. Ausgangspunkt sind dabei einzelne kulturspezifische Dispositionen.

\subsection{Ausdrucksfunktion:}

\section{$<1.4$ Introvertiert - Extrovertiert $>$}

Schon bei geringem Kontakt mit Angehörigen unterschiedlicher Kulturen fällt auf, daß die Bereitschaft, in beliebiger Umgebung offen Gefühle zu zeigen, sehr unterschiedlich ausgeprägt sein kann. $\mathrm{Zu}$ den Regeln britischer Sozialisation gehört u.a.: Feelings are bad manners, womit kaum die Gefühle selbst gemeint sind, sondern die Formen von deren Zurschaustellung. Es geht also gar nicht darum, ob Angehörige unter-schiedlicher Kulturen mehr oder weniger starke Gefühle haben, sondern darum, ob und wie sie diese für andere erkennbar werden lassen. Treffen Mitglieder von Kulturen zusammen, die sich in ihrem offenen Verhalten unter dem Aspekt <introvertiert/extrovertiert> unterscheiden, so kann es zu sehr abweichender gegenseitiger Wahrnehmung kommen: 


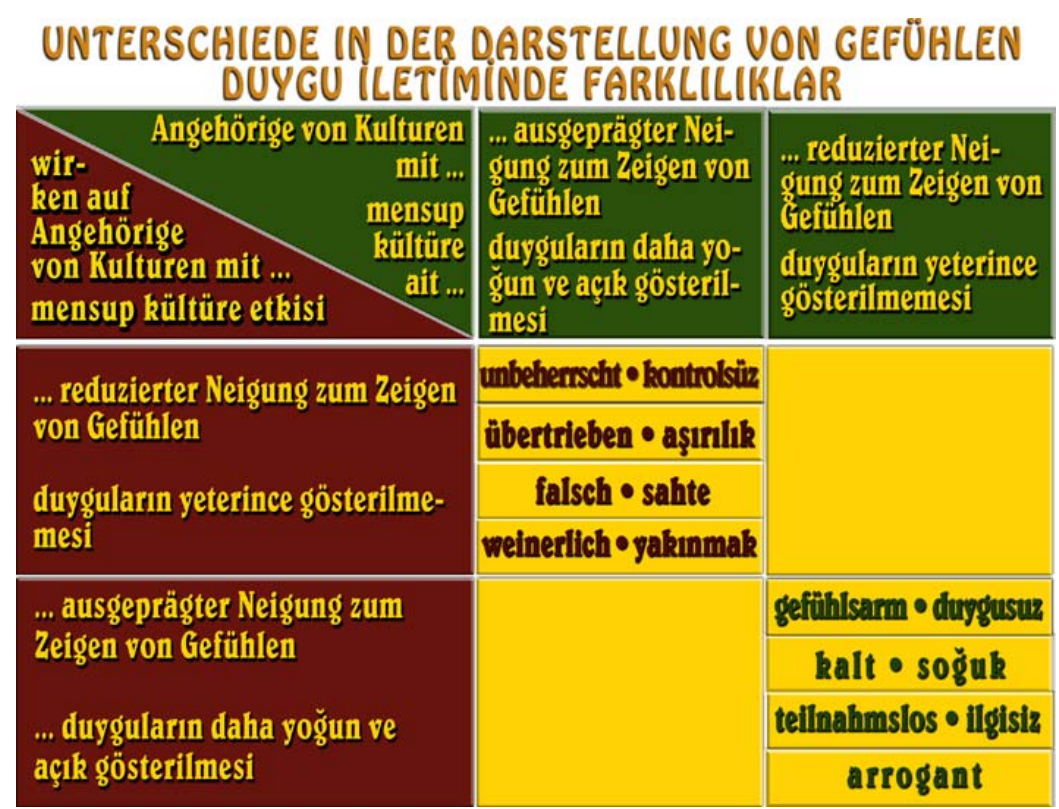

Es läßt sich argumentieren, daß diese Unterschiede mit dem Grad der Modernisierung insofern in Verbindung $\mathrm{zu}$ bringen sind, als die Herausbildung der Eigenständigkeit des Individuums $\mathrm{zu}$ den wesentlichen Merkmalen einer funktionalen Ausdifferenzierung der Gesellschaft gehört. Im Rahmen der Unterscheidung zwischen Gemeinschaft und Gesellschaft, wie sie von TÖNNIES (1887)vertreten wird, ist die Eigenständigkeit des Individuums eine Konsequenz aus der Veregesellschaftung des Menschen (44 ff.). War der Mensch in der Gemeinschaft der Familie oder der Nachbarschaft noch in diese integriert, so bedingt der Übergang zur Gesellschaft die Gegenüberstellung von Individuum und Gesellschaft. Diese Gegenüberstellung hat u.a. das Entstehen einer Grenze zwischen Ich und Gesellschaft zur Folge, die durch die Unterscheidung zwischen Verbergen und Offenbaren (SIMMEL 1908: 259), durch die gleichursprünglichen Bedürfnisse von öffentlicher Geltung und Schamhaftigkeit (PLESSNER 1924: 63) markiert ist; das Zeigen von Gefühlen wird damit zu einem Problem der Grenzüberschreitung. 


\subsection{Appellfunktion:}

\section{$<2.1$ Nähe/Distanz $>$}

Neben den oben schon angesprochenen Anredeformen ist das beobachtbare Raumverhalten der Kommunikationspartner ein Instrument zur Bestimmung von sozialer Nähe/Distanz. Es kann vermutet werden (und alle Erfahrung spricht dafür), daß in allen Kulturen die räumliche Entfernung mit der sozialen korrespondiert. Wenn trotzdem in der Kommunikation von Angehörigen verschiedener Kulturen Unbehagen durch eine irritierende Nähe oder eine befremdliche Distanz entsteht, so kann dies zwei Ursachen haben:

1. Kulturen unterscheiden sich in der räumlichen Distanz des unmarkierten Normalfalls einer zufälligen Begegnung, wobei die Relationen allerdings die gleichen bleiben:

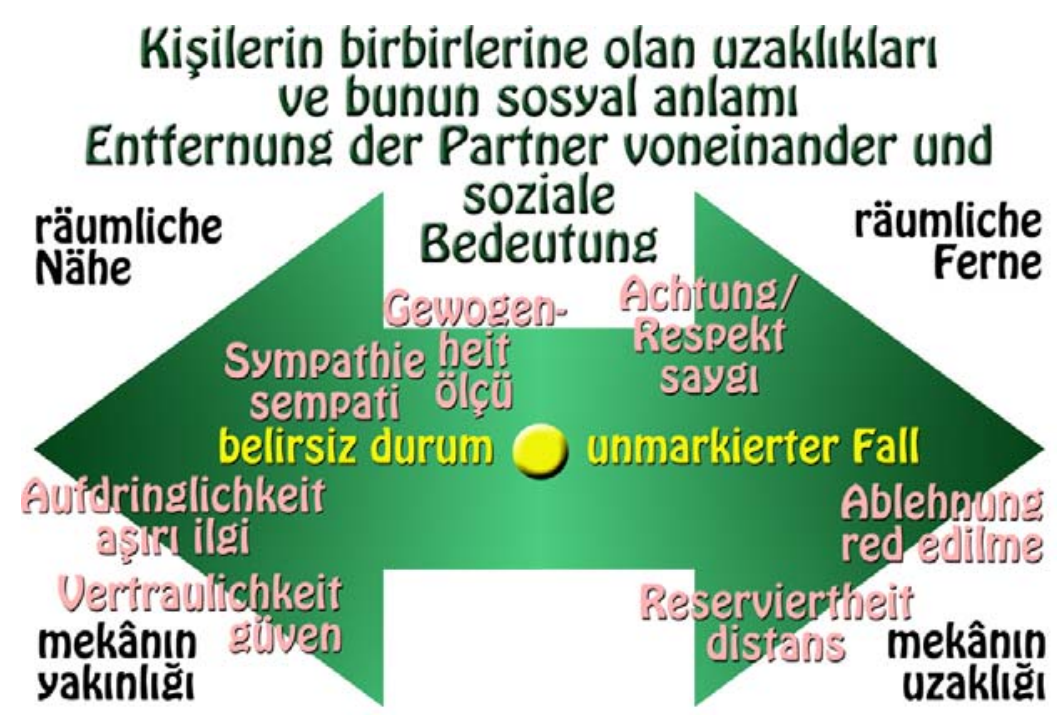

2. Soziale Nähe/Distanz ist in den Kulturen unterschiedlich geregelt. In Gesellschaften, in denen Formen horizontaler Differenzierung eine Rolle spielen wird soziale Nähe bei den Angehörigen einer Gemeinschaft (z.B. einer Familie) vorausgesetzt, während die Nicht-Zugehörigkeit soziale Ferne bedingt; in beiden Fällen von entsprechendem Raumverhalten begleitet. In dem Maße, in dem 
hierarchische Gesichtspunkte von Bedeutung sind, bestimmen Rang- und Standesunterschiede die soziale Nähe/Distanz und damit auch das Raumverhalten. In beiden Fällen sind die Grenzen relativ starr, so daß der Eindruck entstehen kann, daß die meßbare Distanz zwischen Nähe und Ferne größer und weniger variabel ist als in Kulturen, die primär funktional ausdifferenziert sind und in denen die Partner eine größere Freiheit haben, über Nähe/Distanz, sozial wie räumlich, selbst zu entscheiden.

Alles deutet darauf hin, daß das Verhältnis $<$ Nähe/Distanz $>$ auch in seinem beobachtbaren Verhalten mit dem Grad der Modernisierung in Zusammenhang steht, ohne daß von einer mechanischen Abhängigkeit gesprochen werden soll.

\subsection{Darstellungsfunktion:}

\section{$<3.1$ Erlebte/Gemessene Zeit $>$}

$\mathrm{Zu}$ den auffallendsten, bekanntesten und irritierendsten Unterschieden zwischen Angehörigen verschiedener Kulturen gehört das jeweilige Verhältnis zur Zeit, was - auf der Beobachtungsebene - zu Vorwürfen der Unpünktlichkeit bzw. der Pedanterie führt. Die Verbindung zwischen den jeweiligen Zeitvorstellungen und der Modernisierung liegt auf der Hand und ist immer wieder herausgestellt worden (vgl. statt vieler FRASER 1987; DUX 1992).

Zeit an sich ist nicht knapp. Der Eindruck der Zeitknappheit enbtsteht erst aus der Überforderung des Erlebens durch Erwartungen. [...] In einfachen Gesellschaften, in denen jeder im Prinzip alles miterlebt oder doch miterleben könnte, gibt es keine extreme Diskrepanz zwischen der Erwartung objektiven Geschehens und eigenen Erlebens. (LUHMANN 1968: 149)

Folge ist ein gesteigerter Selektionszwang, der in Zeitdruck umgesetzt wird. Statt vom Erleben ist modernes Zeitverständnis nun vom Messen bestimmt. Meßbare Zeit ist als Gundlage des Verhaltens nicht nur angesichts der gesteigerten Coordinationsanforderungen funktional differenzierter Gesellschaft erforderlich (vgl. LUHMANN 1968: 154); sie dient als Maßeinheit für menschliche Leistung und wird damit an das Kommunikationsmedium $<$ Geld $>$ gebunden: Zeit ist Geld - Time is money; an die Stelle des Werkvertrages tritt der an Arbeitszeit gebundene 
Arbeitsvertrag. Indem Zeit, Fristen und Termine eine herausgehobene Bedeutung gewinnen, entsteht eine Zeitideologie: Das Eilige ist das Wichtige: "So kann sich allein aus Zeitproblemen eine Umstrukturierung der Wertordnung ergeben" (LUHMANN 1968: 148).

Aus der Verbindung von Zeitideologie und gesteigerter Komplexität in funktional ausdifferenzierten Gesellschaften folgt ein gesteigerter Planungsbedarf, die Notwendigkeit des Einhaltens von Terminen und daraus - eine umfassende Umstellung von Zweck- auf Routineprogramme (vgl. LUHMANN 1964). Planung, die erwartet, daß sich die Beteiligten auf festgelegte Prioritäten und Abläufe einlassen, und damit auf Termine und Routinen, um diesen Erwartungen zu entsprechen, treffen in weniger funktional differenzierten Gesellschaften auf Vorbehalte oder Unverständnis.

\section{Perspektiven Kultureller Unterschiede}

Kulturelle Unterschiede, so die verbreitete Auffassung, die auch für uns der Ausgangspunkt war, belasten die Beziehungen und speziell die Kommunikation zwischen Menschen. Mit diesem Standpunkt wird jedoch übersehen, daß solche Unterschiede Anregungen geben, die Dinge anders zu sehen, und damit ein Innovations- und Veränderungspotential enthalten. Ebenso wird übersehen, daß kulturelle Unterschiede und damit die Zugehörigkeit zu einer bestimmten Kultur ein wesentlicher Faktor bei der Identitätsbildung der Menschen ist. Das eher verschwommene Selbstbild des Weltbürgers kann nur so lange identitätsstiftend sein, wie es Menschen gibt, die ihre Identität aus der Zugehörigkeit aus einer bestimmten (Landes) kultur beziehen. Nur wer die Bestimmung der Geschichte in einer vorzeitlichen Harmonie aller sieht, kann die Bedeutung der Herausforderungen verkennen, die mit der Verschiedenheit von Kulturen gestellt werden; das wußte man auch in der Folge der Französischen Revolution, die doch Freiheit, Gleichheit und Brüderlichkeit auf ihre Fahnen geschrieben hatte.

Mais enfin, l'histoire n'est pas une idylle, destinée à représenter les hommes perpétuellement heureux, dans des pleines fortunées où coulent des ruissseaux de lait et de miel. (LERMINIER 1832: 192)

Erforderlich ist also weniger die Applanierung aller kulturellen Unterschiede als vielmehr die Entwicklung von Verfahren und 
Verhaltensweisen, die die Kommunikation bei gegebenen Umständen ermöglichen. Die Vision vom global village, wie sie etwa bei MCLUHAN (1964) formuliert ist, bleibt ein Traum und - um ein Bild von Graf MOLTKE zu übernehmen - nicht einmal ein schöner.

Sofern kulturelle Unterschiede in Unterschieden im Prozeß der umfassenden sozialen Evolution begründet sind, ist die Vermutung berechtigt, daß diese Unterschiede im Verlauf der globalen Modernisierung hin zu einer Weltgesellschaft (vgl. LUHMANN 1971; 1997) verschwinden. Dementsprechend ist verschiedentlich von einer Welt- oder Globalkultur die Rede.

Unabhängig von der Wünschbarkeit einer solchen Entwicklung lassen sich eine Reihe von Zweifeln an dieser Prognose vorbringen:

1.Eine Weltkultur hätte keine Grenzen; sie würde nichts ausschließen und wäre mithin gar keine Kultur in einem relevanten Sinne mehr.

2.Ungeklärt ist bislang, wie groß der Anteil jener Unterschiede ist, die wir als genuin bezeichnet haben. Ungeklärt ist bislang auch die Frage, ob und wie diese Unterschiede den Modernisierungsprozeß beeinflussen und unter welchen Bedingungen sie in diesem Prozeß fortbestehen können.

3.Mit der Herausbildung von Funktionssystemen, die keine Grenzen kennen und weltweit operieren, entstehen eigene Kulturen; dies ist schon heute zu beobachten. Die Bereiche Wirtschaft, Wissenschaft und Militär, um einige Beispiele zu nennen, haben bereits heute ein globales Niveau, sodaß etwa nach der politischen Wende in Europa (1989/91) die Mitglieder dieser Funktionssysteme, auch wenn sie unterschiedlichen politischen Systemen angehört hatten, bei ihren Treffen nur geringe Probleme hatten, und zwar nicht nur in bezug auf systemspezifische Anlässe/Themen, sondern auch in den weniger spezifischen Situationen. Es ist also zu vermuten, daß diese Systeme unterschiedliche Kulturen entstehen lassen, die umfassender sind als die, die sich aus der Mitgliedschaft in einem Funktionssystem herleiten lassen. In einem gewissen Umfang vermögen Funktionssysteme auch, Identitätsangebote bereitzustellen. In der Herausbildung und Globalisierung von Funktionssystemen ist also mit dem Entstehen von globalen Systemkulturen zu rechnen. 
4. Die Herausbildung von Funktionssystemen hat eine Abschottung dieser Systeme zur Folge, die durch das Entstehen von Netzwerken als Inklusionssystemen zu kompensieren versucht wird (vgl. LUHMANN 1995). Solche Netzwerke können kulturell begründet werden und auch eigene Kulturen begründen.

5. In funktional ausdifferenzierten Gesellschaften ist der einzelne Mitglied in mehreren Teilsystemen und Netzwerken, so daß Patchworkoder Hybridkulturen entstehen: "Was als Person sichtbar ist, ist eigentlich nur eie Erwartungskollage, das, was man notfalls und normalerweise zusammenhält, wenn man mit anderen zu tun hat" (LUHMANN 1985: 268). Einen theoretischen Rahmen hat Dirk BAECKER (2000) unter dem Stichwort der Meta-Kultur vorgelegt.

Modernisierung und Globalisierung bedeuten - so die weiter zu verfolgende Hypothese - nicht das Ende aller kulturellen Unterschiede. Dieser Beitrag ist als Forschungsprogramm gedacht, um weitere Klarheit zu gewinnen.

\section{Verwendete Literatur}

Baecker, Dirk (2000); Wozu Kultur?, Berlin: Kulturverlag Kadmos.

Bismarck, Otto v. (1898): Gedanken und Erinnerungen, 2 Bände, Stuttgart: J.G. Cotta Nachf, 1898.

Bolz, Norbert (2002); Das konsumistische Manifest, München: Wilhelm Fink.

Dux, Günter (1992); Die Zeit in der Geschichte. Ihre Entwicklungslogik vom Mythos zur Weltzeit, stw 1025, Frankfurt a.M.: Suhrkamp.

Elias, Norbert (1969a); Über den Prozeß der Zivilisation. Soziogenetische und psychogenetische Untersuchungen. Erster Band: Wandlungen des Verhaltens in den weltlichen Oberschichten des Abendlande, stw 158, 2. Aufl., Frankfurt a.M.: Suhrkamp 1977.

Elias, Norbert (1969b); Über den Prozeß der Zivilisation. Soziogenetische und psychogenetische Untersuchungen. Zweiter Band: Wandlungen der Gesellschaft. Entwurf zu einer Theorie der Zivilisation, stw 159. 2. Aufl., Frankfurt a.M.: Suhrkamp 1977. 
Fraser, Julius T. (1987); Die Zeit. Auf den Spuren eines vertrauten und doch fremden Phänomens, dtv 11422, München: dtv 1991.

Frazer, James George (1922); The Golden Bough. A Study in Magic and Religion, Abridged edition, London: Macmillan 1987.

Glissant, Édouard (1999); Traktat über die Welt, Heidelberg: Wunderhorn.

Gontscharow, Iwan (1859); Oblomow, Roman, 2. Aufl., Zürich: Manesse 1987.

Hobbes, Thomas (1651); Leviathan, oder Stoff, Form und Gewalt eines kirchlichen und bürgerlichen Staates, Hg. Iring FETSCHER, stw 462, Frankfurt a.M.: Suhrkamp 1984.

Hofstätter, Peter R. (1957); Fischer Lexikon Psychologie "Völkerpsychologie", FL 6., Neuausg. (Frankfurt a.M.: Fischer 1977), ss. $343-347$.

Hofstede, Geert (1984); Culture's Consequences. International Differences in Work-Related Values, Cross-Cultural Research and Methodology Series 5. London/\&c.: Sage 1984.

Hofstede, Geert (1991a); Cultures and Organizations. Software of the Mind, New York/\&c.: McGraw-Hill, 1997.

Hofstede, Geert (1991b); Lokales Denken, globales Handeln. Kulturen, Zusammenarbeit und Management., [engl. Original: Cultures and Organizations, Software of the Mind] dtv 50807, München: dtv 1997.

Jakobson, Roman (1960); "Linguistics and Poetics", Style in Language, Hg. Thomas A. Sebeok (Repr., Cambridge, Mass.: MIT Press 1968), ss. 350 - 377.

Krusche, Helmut (1992); Der Frosch auf der Butter. NLP - Die Grundlagen des Neuro-Linguistischen Programmierens, 2. Aufl., Düsseldorf/etc.: Econ 1994.

Lange, Wolfgang (1983); "Tod ist bei Göttern nur ein Vorurteil. Zum Komplex des Mythos bei Nietzsche", Mythos und Moderne. Begriff und Bild einer Rekonstruktion., Hg. Karl Heinz BOHRER. es 1144 (Frankfurt: Suhrkamp 1983), ss. 111 - 137. 
Leggewie, Claus (2000); "Hybridkulturen", Europa oder Amerika? Zur Zukunft des Westens, Merkur Sonderheft 617/618, ss. 878 - 889.

Lerminier, E. (1832) ; Philosophie du droit. 3. Aufl., Paris: Librairie de Guillaumin, 1853.

Levi-Strauss, Claude (1958); Strukturale Anthropologie, Frankfurt a.M.: Suhrkamp 1967.

Lübbe, Hermann (1973); "Was heißt: <Das kann man nur historisch erklären>?", Geschichte - Ereignis und Erzählung, Hgg. Reinhart Koselleck/Wolf-Dieter Stempel, Poetik und Hermeneutik V (München: Fink 1973), ss. $542-554$.

Luhmann, Niklas (1964); "Lob der Routine", N.L.: Politische Planung. Aufsätze zur Soziologie von Politik und Verwaltung (2. Aufl., Opladen: Westdeutscher Verlag 1975), ss. 113 - 142. (zuerst: Verwaltungsarchiv 55 (1964), ss. 1 - 33.).

Luhmann, Niklas (1968); "Die Knappheit der Zeit und die Vordringlichkeit des Befristeten", N.L.: Politische Planung. Aufsätze zur Soziologie von Politik und Verwaltung (2. Aufl., Opladen: Westdeutscher Verlag 1975), ss. 143 - 164. (zuerst: Die Verwaltung 1 (1968), ss. 3 - 30.)

Luhmann, Niklas (1971); "Die Weltgesellschaft". N.L.: Soziologische Aufklärung 2. Aufsätze zur Theorie der Gesellschaft (Opladen: Westdeutscher Verlag 1975), ss. 51 - 71.

Luhmann, Niklas (1985); "Die Soziologie und der Mensch", N.L.: Soziologische Aufklärung 6. Die Soziologie und der Mensch (Opladen: Westdeutscher Verlag 1985), ss. 265 - 274. (zuerst: Neue Sammlung 25 (1985), ss. 33 - 41.)

Luhmann, Niklas (1986); "<Distinctions directrices>, Über Codierung von Semantiken und Systemen", N.L.: Soziologische Aufklärung 4. Beiträge zur funktionalen Differenzierung der Gesellschaft (Opladen: Westdeutscher Verlag 1987), ss. 13 - 31.

Luhmann, Niklas (1995); "Inklusion und Exklusion", N.L.: Soziologische Aufklärung 6. Die Soziologie und der Mensch (Opladen: Westdeutscher Verlag 1995), ss. 237 - 264. 
Luhmann, Niklas (1997); Die Gesellschaft der Gesellschaft, 2 Teilbände, Frankfurt a.M.: Suhrkamp.

McLuhan, Marshall (1964); Understanding Media: The Extensions of Man, 9th. pr., New York: Signet.

Marquard, Odo (1973); "Schwierigkeiten mit der Geschichtsphilosophie", O.M.: Schwierigkeiten mit der Geschichtsphilosophie. Aufsätze. stw 394 (Frankfurt a.M.: Suhrkamp 1983), ss. 13 - 33.

Pike, Kenneth L. (1967); Language in Relation to a Unified Theory of the Structure of Human Behavior, Janua Linguarum, series maior 24. 2nd., rev. ed.., The Hague/Paris: Mouton.

Plessner, Helmuth (1924); "Grenzen der Gemeinschaft. Eine Kritik des sozialen Radikalismus", H.P.: Gesammelte Schriften V: Macht und menschliche Natur. Hgg. Günter Dux / Odo Marquard / Elisabeth Ströker (Frankfurt a.M.: Suhrkamp 1981), ss. 7 - 133.

Sander, Uwe (1998); Die Bindung der Unverbindlichkeit. Mediatisierte Kommunikation in modernen Gesellschaften, es 2042, Frankfurt a.M.: Suhrkamp.

Schiller, Friedrich v. (1789); "Was heißt und zu welchem Ende studirt man Universalgeschichte? Eine akademische Antrittsrede", F.v. SCH.: Sämmtliche Werke mit Stahlstichen. Zehnter Band (Stuttgart/Tübingen: Cotta 1836), ss. 414 - 442.

Simmel, Georg (1908); Soziologie. Untersuchungen über die Formen der Vergesellschaftung, 5. Aufl., Berlin: Duncker \& Humblot 1968.

Thomas, Alexander (1993); "Entwicklungslinien und Erkenntniswert kulturvergleichender Psychologie", Kulturvergleichende Psychologie, Eine Einführung. Hg. Alexander Thomas (Göttingen/\&c.: Hogrefe 1993), ss. $27-51$.

Tönnies, Ferndinand (1887); Gemeinschaft und Gesellschaft. Grundbegriffe der reinen Soziologie, 3.,unv. Aufl., Darmstadt: WB 1991.

Van Gennep, Arnold (1909); Übergangsriten (Les rites de passage), Frankfurt/New York/Paris: Campus/Maison des Sciences de l'Homme 1986. 